\title{
Cardioprotective Effects of Oral Trimetazidine in Diabetic Patients With Anterior Wall Myocardial Infarction Treated with Thrombolysis
}

\author{
Mohamed Shehata
}

\begin{abstract}
Background: Trimetazidine is an anti-ischemic agent with antioxidant activity. This study sought to evaluate the impact of oral trimetazidine on extent of myocardial damage in diabetic patients who were presented with anterior wall ST-segment elevation myocardial infarction (STEMI).
\end{abstract}

Methods: One hundred patients were prospectively enrolled, and then randomly assigned to receive oral trimetazidine $(70 \mathrm{mg}$ then $35 \mathrm{mg}$ bid) (group A, 50 patients) or placebo (group B, 50 patients), starting before thrombolysis. Serum creatine kinase-T and MB (CK-T and CK-MB) were measured serially. Degree of ST-segment resolution was recorded after 90 minutes. Left ventricle ejection fraction (LVEF) was assessed at baseline and after 6 months. Adverse events were recorded after thrombolysis and 6 months later.

Results: Mean age of the study cohort was $59.05 \pm 3.8$ years (males: $60 \%)$. After 24 hours, $45(90 \%)$ patients in group A vs. $10(20 \%)$ patients in group B showed peaking of CK-T and CK-MB levels $(\mathrm{P}<0.05)$. Both biomarkers' levels were significantly higher in the placebo group at different sampling times. Complete resolution of ST-segment elevation was recorded in $35(70 \%)$ patients in group A vs. $18(36 \%)$ patients in group $\mathrm{B}(\mathrm{P}<0.05)$. Six months later, group A showed higher LVEF and fewer cardiac adverse events $(\mathrm{P}$ $<0.05)$.

Conclusion: In diabetic patients receiving thrombolytic therapy for anterior wall STEMI, oral trimetazidine dosing was associated with less myocardial damage, earlier successful reperfusion, improvement of LVEF and less cardiac adverse events.

Keywords: Trimetazidine; Thrombolysis; Diabetes mellitus; Cardiac enzymes

\footnotetext{
Manuscript accepted for publication April 11, 2014

Department of Cardiology, Faculty of Medicine, Ain Shams University, Cairo, Egypt. Email: smarttmann@hotmail.com
}

doi: http://dx.doi.org/10.14740/cr330w

\section{Introduction}

Trimetazidine (1-(2, 3, 4-trimethoxybenzyl) piperazine) is a cellular anti-ischemic agent that selectively inhibits the activity of the final enzyme of the fatty acid oxidation pathway, 3-ketoacylcoenzyme A thiolase. Administration of this drug leads to a switch in preference of the energy substrate, resulting in partial inhibition of fatty acid oxidation and increased glucose oxidation. Clinical studies had shown that trimetazidine has cardio-protective effects in the setting of myocardial ischemia including acute myocardial infarction (MI) [1-6]. Trimetazidine has been described as a cellular anti-ischemic agent [7]. Thrombolytic agents are highly effective in re-opening occluded coronary arteries and it is the accepted mechanism by which the myocardium is preserved and thus lives saved [8]. However, within few hours of the administration of a thrombolytic agent or placebo, there are more deaths in the active group than in the control. This is attributed to reperfusion injury, as suggested by experiments using animal models. The prognosis of patients with acute MI receiving thrombolytic therapy may be improved by treatment that could reduce the production or increase the elimination rate of free radicals [5]. It was demonstrated that trimetazidine prevents the deleterious effects of ischemia, reperfusion at both the cellular and the mitochondrial levels, and exerts potent antioxidant activity on various tissue preparations $[9,10]$. Trimetazidine inhibits the excessive release of oxygen-free radicals, increases glucose metabolism, limits intracellular acidosis, protects adenosine triphosphate (ATP) stores, reduces membrane lipid peroxidation and inhibits neutrophil infiltration after ischemia-reperfusion [11, 12].

This study sought to evaluate the impact of trimetazidine oral loading on extent of myocardial damage (cardiac enzymes release) and success of reperfusion of the infarctrelated artery (ST-segment resolution), in diabetic patients who were presented with anterior wall ST-segment elevation myocardial infarction (STEMI). Also, the impact of continued oral therapy on left ventricle (LV) systolic function, morbidity and mortality was evaluated after a 6-month follow-up period. 


\section{Methods}

\section{Study design and data collection}

One hundred consecutive diabetic patients, carrying the diagnosis of anterior wall STEMI, were prospectively enrolled in this study. They were referred to the coronary care unit (CCU) in the period between January 2011 and July 2013. All patients received thrombolytic therapy within $6 \mathrm{~h}$ of onset of chest pain. Exclusion criteria included: prior history of acute coronary syndrome, cardiogenic shock, prior history of percutaneous coronary intervention (PCI) or coronary artery bypass graft surgery, congenital heart disease or any myocardial disease apart from ischemia, skeletal muscle disorders, limited life expectancy due to coexistent disease, for example malignancy, previous treatment with trimetazidine or presence of contraindications to oral trimetazidine use (for example, Parkinson's disease and other motion disorders) [13] or thrombolytic therapy (for example, previous intracranial hemorrhage or recent major trauma or surgery) [14]. After enrollment and before thrombolysis, patients were randomly assigned in 1:1 fashion to either trimetazidine group (group A) or placebo group (group B) according to a computer-generated random series of numbers. Randomization was performed by block randomization (blocks of 10 patients). Group A patients received oral trimetazidine loading dose (70 mg) upon CCU admission (15 - $30 \mathrm{~min}$ before receiving thrombolytic therapy), followed by daily dosing (35 mg twice daily), continued for 6 months. Group B received a placebo formula for the same period of time. Physicians and medical staff in CCU were unaware of block randomization. All included patients were subjected to detailed history taking including drug-intake, baseline followed by serial 12 lead electrocardiogram (ECG) and transthoracic echocardiogram (TTE). All patients received fibrin-specific thrombolytic (fibrinolytic) therapy in the form of intravenous (IV) alteplase (tPA), administered as follows: a bolus of $15 \mathrm{mg}$, followed by $0.75 \mathrm{mg} / \mathrm{kg}$ over $30 \mathrm{~min}$ (up to $50 \mathrm{mg}$ ) and then $0.5 \mathrm{mg} / \mathrm{kg}$ over $60 \mathrm{~min}$ (up to $35 \mathrm{mg}$ ) [14]. Before inclusion, informed written consent was obtained from each patient and the study protocol was reviewed and approved by our local institutional human research committee, as it conforms to the ethical guidelines of the 1975 Declaration of Helsinki, as revised in 2008 .

\section{Definition of risk factors of coronary artery disease}

The presence of hypertension was defined as systolic blood pressure $\geq 140 \mathrm{~mm} \mathrm{Hg}$ and/or diastolic blood pressure $\geq 90$ $\mathrm{mm} \mathrm{Hg}$, previously recorded by repeated non-invasive office measurements, which led to life-style modification and/or intake of antihypertensive drug therapy [15]. Dyslipidemia was defined as LDL cholesterol $>100 \mathrm{mg} / \mathrm{dL}$, and/or serum triglycerides $>150 \mathrm{mg} / \mathrm{dL}$, and/or HDL cholesterol $<40 \mathrm{mg} /$
$\mathrm{dL}(<50 \mathrm{mg} / \mathrm{dL}$ in women $)[16]$.

\section{Baseline echocardiographic assessment}

Assessment of regional and global LV systolic functions was performed in all patients by TTE using a General Electric Vivid 7 cardiac ultrasound machine (General Electric, Horten, Norway), equipped with harmonic imaging capabilities. A 2.5-MHz phased array probe was used to obtain standard 2D, M-mode and Doppler images. Patients were examined in the left lateral recumbent position using standard parasternal and apical views. Left ventricle ejection fraction (LVEF) by modified Simpson's method, LV end diastolic volume (LVEDV), LV end systolic volume (LVESV) using bi-plane algorithm, wall motion abnormalities and wall motion score index (WMSI) were recorded for every patient, 24 - $48 \mathrm{~h}$ after thrombolysis and 6 months later. Regional wall motion was assessed according to the standard 17-segment model as recommended by the American Society of Echocardiography [17]. WMSI was calculated by dividing the sum of scores of the whole LV segments divided by the number of scored segments. Images were digitized in cine-loop format, and saved for subsequent playback and analysis. Views were analyzed by a single echocardiographer employing the software program of the echocardiography machine and blinded to the study protocol. Regional wall motion abnormalities were visually assessed for each segment individually, considering both endocardial excursion and systolic thickening. Each segment was graded according to the semi-quantitative scoring system described by Cerqueira et al [17]. Segments with poorly defined endocardial borders for $50 \%$ or more of their length were considered non-visualized and assigned a score of 0 [18]. Wall thickening was assessed at a distance of at least $1 \mathrm{~cm}$ from the adjacent segment, to minimize the effect of tethering [19]. Images were digitized in cine-loop format, and saved for subsequent playback and analysis. Views were analyzed by a single echocardiographer (for both baseline and follow-up assessments) employing the software program of the echocardiography machine and was blinded to the study protocol.

\section{Medical treatment and laboratory work-up}

Blood sampling for creatine kinase-total (CK-T) and creatine kinase-MB (CK-MB) isoenzyme levels was done just before thrombolysis and then repeated after $12 \mathrm{~h}$ followed by daily sampling till normalization of serum levels of both markers. Measurements were performed with the use of a chemiluminescence immunoassay and Elecsys 2010 analyzer from Roche Diagnostics Laboratory. The upper limit of normal reference range was $220 \mathrm{U} / \mathrm{L}$ for $\mathrm{CK}-\mathrm{T}$ and $8.8 \mathrm{U} / \mathrm{L}$ for CK-MB.

All patients received regular anti-ischemic medical treatment including: aspirin, clopidogrel, beta-blockers, an- 
Table 1. Baseline Characteristics of the Two Study Groups

\begin{tabular}{llll}
\hline Variable & Group A $(\mathbf{n}=\mathbf{5 0})$ & Group B $(\mathbf{n}=\mathbf{5 0})$ & P value* $^{*}$ \\
\hline Age (years) & $59.6 \pm 5.4$ & $58.5 \pm 2.3$ & $>0.05$ \\
Males & $31(62)$ & $29(58)$ & $>0.05$ \\
Hypertension & $24(48)$ & $25(50)$ & $>0.05$ \\
Dyslipidemia & $20(40)$ & $21(42)$ & $>0.05$ \\
Smoking & $15(30)$ & $17(34)$ & $>0.05$ \\
Baseline CK-T (U/L) & $222 \pm 8.4$ & $218 \pm 6.4$ & $>0.05$ \\
Baseline CK-MB (U/L) & $8.9 \pm 2.4$ & $9.3 \pm 3.6$ & $>0.05$ \\
HbAlc (\%) & $7.2 \pm 0.2$ & $7.1 \pm 0.3$ & $>0.05$ \\
\hline
\end{tabular}

CK-T: creatine kinase-total; CK-MB: creatine kinase-MB isoenzmye; HbA1c: glycated hemoglobin. Categorical variables are presented as number (percentage). Continuous variables are presented as mean \pm standard deviation. ${ }^{*}$ Pearson Chisquare and Student's $t$-test.

giotensin converting enzyme inhibitors and nitrates. All patients received enoxaparin upon admission ( $30 \mathrm{mg}$ IV bolus followed by $1 \mathrm{mg} / \mathrm{kg} / 12 \mathrm{~h}$, given subcutaneously) [14]. They were followed up closely for recording of post-MI complications (pump failure, arrhythmias, mechanical complications, LV thrombus, and so on), if any.

\section{Twelve-lead ECG monitoring}

A 12-lead ECG was recorded upon presentation to CCU (baseline ECG), 90 min after thrombolysis and at $12 \mathrm{~h}$ intervals throughout the CCU stay period. Additionally, three- lead ECG monitoring was constantly followed up especially for occurrence of malignant arrhythmias. ST-segment resolution was judged using the ECG done $90 \mathrm{~min}$ after thrombolysis. The sum of ST-segment elevation $20 \mathrm{~ms}$ after the $\mathrm{J}$ point was calculated and compared with the baseline ECG. The percent resolution was categorized as complete $(\geq 70 \%)$, partial $(30 \%$ to $<70 \%)$, or none $(<30 \%)[20]$.

\section{Recording of clinical adverse events}

In-hospital adverse events were recorded for both study groups including: in-hospital mortality, heart failure, ma-

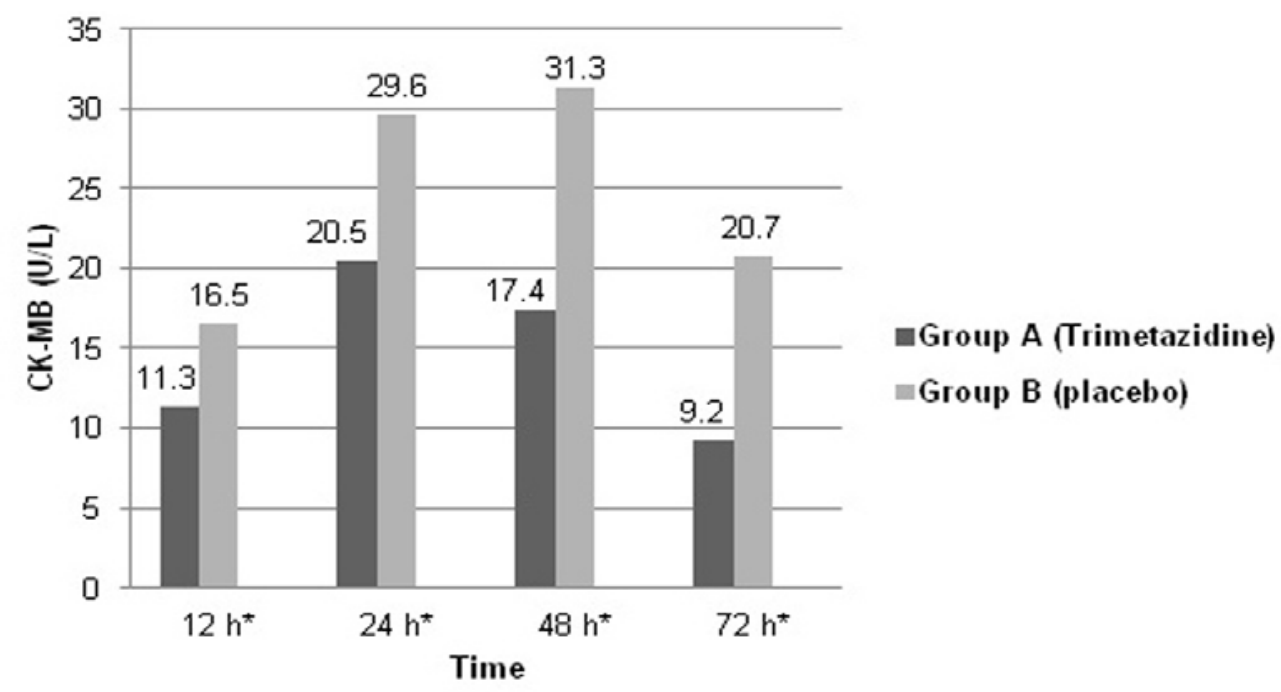

Figure 1. Graphic presentation showing changes in mean CK-MB levels in both study groups in the first $72 \mathrm{~h}$ after thrombolysis. ${ }^{*} \mathrm{P}<0.05$ (Student's $t$-test). 


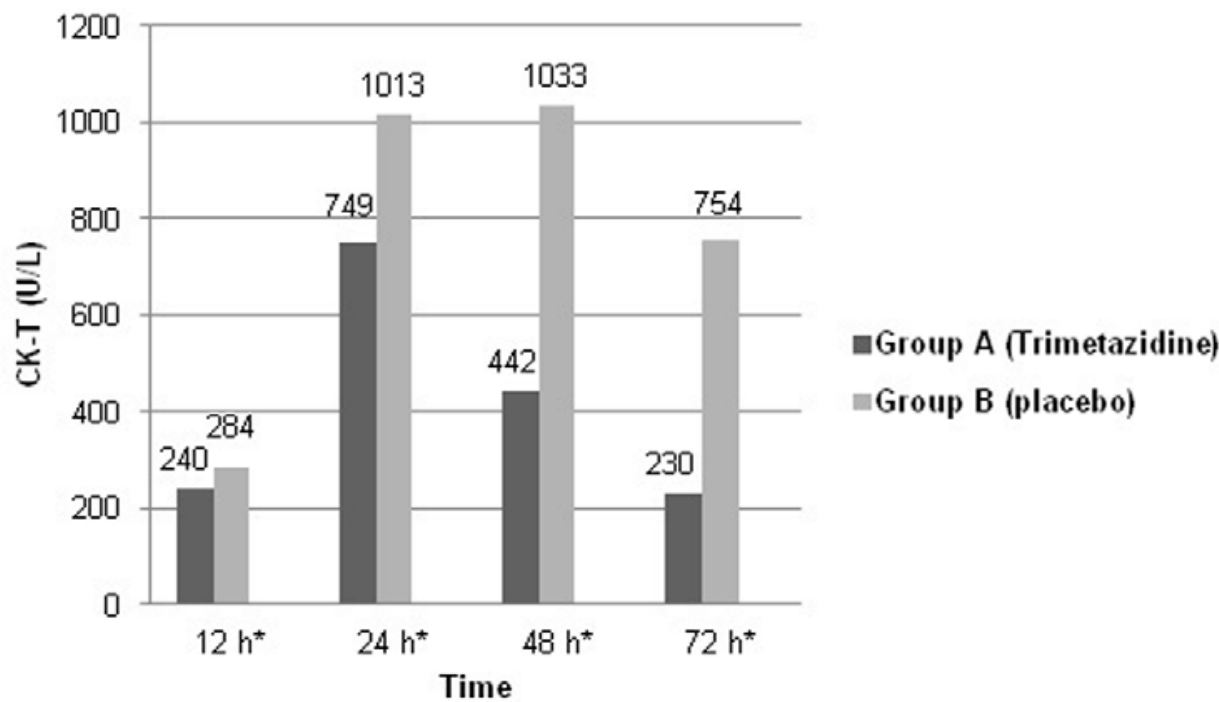

Figure 2. Graphic presentation showing changes in mean CK-T levels in both study groups in the first $72 \mathrm{~h}$ after thrombolysis. ${ }^{*} \mathrm{P}<0.05$ (Student's $t$-test).

lignant ventricular arrhythmias, ventricular septal rupture, occurrence of LV thrombus, recurrence of angina pain, reinfarction and cerebrovascular stroke. Six months' data collection included recording of cardiac mortality, hospitalization due to heart failure and re-infarction.

\section{Statistics}

All continuous variables were statistically described in terms of mean \pm standard deviation (SD). Categorical variables were described with absolute and relative (percentage) frequencies. Comparison of continuous variables between the study groups was done using Student's $t$-test. For comparing categorical data, Pearson Chi-square and Fisher exact tests were performed. $P$ values were used to describe significance. All statistical calculations were done using Statistical Package for Social Sciences (SPSS for Windows) software (version 15.0, SPSS Inc., Chicago, IL, USA).

\section{Results}

\section{Baseline clinical characteristics}

A total of 100 consecutive diabetic patients with anterior wall STEMI were prospectively enrolled in this study. All patients received IV alteplase, in addition to adjunctive antithrombotic therapy and anti-ischemic therapy. The current study comprises 50 patients randomly assigned to trimetazidine group (group A) receiving the drug according to the previously described dosing regimen, and 50 others randomly assigned to the placebo group (group B). The mean age of the whole study cohort was $59.05 \pm 3.8$ years, $60(60 \%)$ be- ing male patients. The two study groups were matched regarding age, gender, risk factors of coronary artery disease and baseline TTE data. No statistically significant difference was found between the two groups concerning mean baseline glycated hemoglobin (HbAlc) level. Table 1 shows baseline characteristics of the two study groups.

\section{Cardiac enzymes and ST-segment resolution}

Mean baseline CK-T and CK-MB levels were not significantly different between both study groups. After $24 \mathrm{~h}, 45$ $(90 \%)$ patients in group A versus $10(20 \%)$ patients only in group B showed peaking of CK-T and CK-MB levels (P < 0.05 ). Also, both biomarkers' levels were recorded to be significantly higher in the placebo group at different sampling times. Patients in group A showed almost normalized cardiac enzymes levels after $72 \mathrm{~h}$ (mean CK-MB: $9.2 \pm 1.2$; CK-T: $230 \pm 6.5 \mathrm{U} / \mathrm{L}$ ), while group B patients almost reached enzymes' normalization after $96 \mathrm{~h}$ (mean CK-MB: $9.5 \pm 2.3$ U/L; CK-T: $228.3 \pm 6.4$ U/L). Mean CK-T and CK-MB levels recorded in the first $72 \mathrm{~h}$ after thrombolysis are shown in Figures 1 and 2. ECG done 90 min after thrombolysis revealed complete resolution of ST-segment elevation in $35(70 \%)$ patients in group A, while this was recorded in $18(36 \%)$ patients only in group B $(\mathrm{P}<0.05)$. ST-segment changes in both study groups are shown in Figure 3.

\section{Echocardiographic data}

Echocardiographic data obtained within $24-48 \mathrm{~h}$ after thrombolysis did not differ significantly between both study groups. However, after 6 months, group A showed a highly significant improvement in LVEF, compared to baseline 


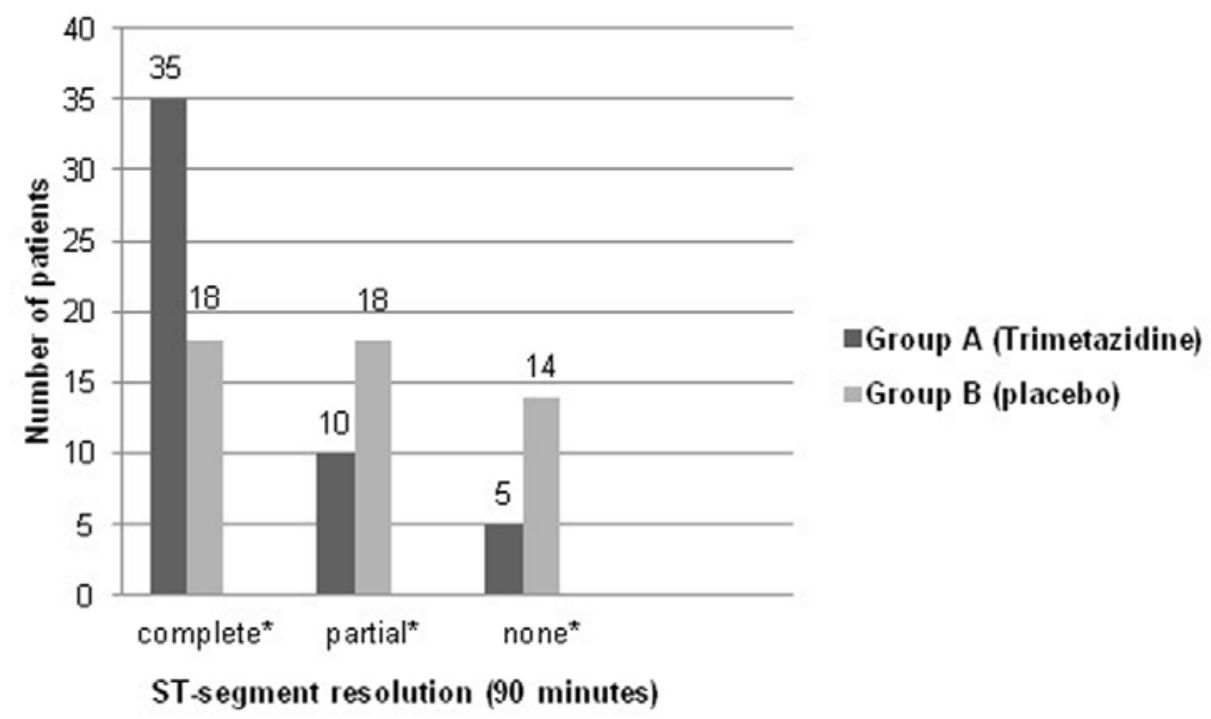

Figure 3. Graphic presentation showing ST-segment changes (90 min after thrombolysis) in both study groups. * $\mathrm{P}<0.05$ (Pearson Chi-square test).

data. Also, there was a significant reduction in LVESV and WMSI. Echocardiographic data collection was done for 47 patients only in group B (three cases of cardiac mortality were recorded during the follow-up period). Analysis of intra-observer variability revealed a close correlation between repeated recordings of regional wall motion by the single operator, with a correlation coefficient $r$ of 0.91 . Echocardiographic data are summarized in Table 2.

\section{Clinical adverse events (in-hospital and follow-up)}

There were no recorded cases of mortality or mechanical

Table 2. Echocardiographic Data of Both Study Groups (24 - 48 h After Thrombolysis and 6 Months Later)

\begin{tabular}{clll}
\hline & Baseline & After 6 months & P value* \\
& & & \\
\hline Group A $(\mathrm{n}=50)$ & & $39.5 \pm 5.9$ & $<0.001$ \\
LVEF $(\%)$ & $33.1 \pm 4.5$ & $181.8 \pm 13$ & $>0.05$ \\
LVEDV $(\mathrm{mL})$ & $183 \pm 12.7$ & $110.2 \pm 13$ & $<0.05$ \\
LVESV $(\mathrm{mL})$ & $121.8 \pm 9.2$ & $1.29 \pm 0.2$ & $<0.05$ \\
WMSI & $1.64 \pm 0.3$ & & $>0.05$ \\
Group B $(\mathrm{n}=47)$ & & $33.6 \pm 3.4$ & $>0.05$ \\
LVEF $(\%)$ & $33.4 \pm 3.5$ & $180.7 \pm 18$ & $>0.05$ \\
LVEDV $(\mathrm{mL})$ & $181 \pm 19.9$ & $110 \pm 22$ & $>0.05$ \\
LVESV $(\mathrm{mL})$ & $113.7 \pm 11.7$ & $1.64 \pm 0.4$ & \\
WMSI & $1.7 \pm 0.3$ & &
\end{tabular}

LVEF: left ventricle ejection fraction, LVEDV: left ventricle end diastolic volume; LVESV: left ventricle end systolic volume; WMSI: wall motion score index. All variables are presented as mean \pm standard deviation. ${ }^{*}$ Student's $t$-test. 
Table 3. Recorded Adverse Events for Both Study Groups (During In-Hospital Stay and After 6 Months)

\begin{tabular}{llll}
\hline Variable & Group A $(\mathbf{n}=\mathbf{5 0})$ & Group B (n= 50) & P value* \\
\hline In-hospital & & & $>0.05$ \\
HF & $5(10)$ & $7(14)$ & $>0.05$ \\
Sustained VA & $4(8)$ & $5(10)$ & $>0.05$ \\
LV thrombus & $4(8)$ & $6(12)$ & $>0.05$ \\
Recurrence of angina & $8(16)$ & $6(12)$ & $>0.05$ \\
Re-infarction & 0 & $2(4)$ & $>0.05$ \\
Cerebrovascular stroke & 0 & $1(2)$ & $<0.05$ \\
Six months & & $3(6)$ & $<0.05$ \\
Cardiac mortality & 0 & $10(20)$ & $<0.05$ \\
Hospitalization for HF & $3(6)$ & $5(10)$ & \\
Re-infarction & $1(2)$ & &
\end{tabular}

HF: heart failure; VA: ventricular arrhythmia; LV: left ventricle. All variables are presented as number (percentage). *Pearson Chi-square and Fisher exact tests.

complications during the in-hospital stay among patients in both study groups. There was no significant difference between both groups concerning incidence of adverse events during hospitalization. However, data recording after 6 months revealed higher incidence of cardiac mortality, mostly sudden cardiac death, hospitalization for heart failure and re-infarction (managed medically) in group $\mathrm{B}(\mathrm{P}<0.05)$. These data are summarized in Table 3. All patients were followed up regularly through cardiology out-patient clinic and phone calls with adjustments of medical treatment, whenever needed. Glycated hemoglobin was checked at the end of the follow-up period with no significant difference between both groups (group A: $6.9 \pm 0.22 \%$, group B: $7 \pm 0.12, \mathrm{P}>0.05$ ).

\section{Discussion}

The current study presented two points of strength concerning pre-treatment with oral trimetazidine in diabetic patients, receiving thrombolytic therapy as a reperfusion strategy for management of STEMI. The first point was shown when the group of patients pretreated with trimetazidine showed significantly less myocardial damage evidenced by earlier peaking and lower total amount of cardiac enzymes' (CK-T and $\mathrm{CK}-\mathrm{MB}$ ) release during the hospitalization period. Furthermore, the same group of patients (continued trimetazidine therapy for 6 months) showed significant myocardial function recovery evidenced by significant improvement of
LVEF after 6 months. Interestingly, that was associated with less incidence of cardiac mortality, hospitalization for heart failure and repeated MI. The daily dose of trimetazidine used in the current study (35 mg twice daily) was reviewed by European Medicines Agency in June 2012 and was reported to improve ischemic symptoms [13]. The results of the current study add evidence to the safety of this daily dose. However, the conventionally used dose is $20 \mathrm{mg}$ thrice daily. The author sought to select patients with anterior wall STEMI for inclusion, in order to obtain more pronounced results especially concerning cardiac enzymes' levels and LVEF change.

It is worth highlighting that both groups of patients showed a comparable baseline risk profile. Moreover, there was no significant difference recorded regarding baseline CK-T and CK-MB levels. Also, both groups seemed to have a comparable glycemic control evidenced by almost similar mean baseline HbA1c levels. The favorable effect of trimetazidine on myocardial necrosis could be explained by both its metabolic and biological effects. Trimetazidine had been shown to act as a cellular anti-ischemic agent without any hemodynamic effects [21]. It acts by improving cardiac energy metabolism by switching ATP production from lipid to glucose oxidation, thus enhancing intra-mitochondrial coupling and favoring a more efficient mode of ATP production per mole of oxygen. Moreover, trimetazidine reduces intracellular acidosis and protects against oxygen free radical induced toxicity. The drug therefore directly protects myocyte structure and function and increases cell resistance to hypoxic 
stress [11, 22, 23]. The potent antioxidant effect of trimetazidine had been demonstrated in minimizing myocardial, renal and hepatic ischemia-reperfusion injury [24-26]. Moreover, it was shown that pre-treatment with trimetazidine was effective in reducing the size of the infarct that develops in the blood-perfused rabbit model of myocardial ischemia [27]. Another animal experiment demonstrated that trimetazidine could limit lethal ischemia-reperfusion injury by inhibiting mitochondrial permeability transition pore opening, which represents a crucial event in cardiomyocyte death following myocardial ischemia-reperfusion [28].

The pattern of cardiac biomarkers release in both study groups declared that trimetazidine group of patients exhibited less myocardial damage and put the cardioprotective effect of this drug in a clear place to recognize. This appeared to be also related to earlier successful reperfusion of the infarcted myocardium. Evidence was added upon recording that the vast majority of trimetazidine group of patients showed complete ST-segment resolution 90 min after receiving the fibrinolytic therapy.

Although, data recording concerning in-hospital adverse events revealed no significant difference between both study groups, the question was: how about an extended follow-up period for myocardial systolic function and clinical adverse events? The answer after 6 months interestingly revealed a significant improvement of LV systolic function in trimetazidine group of patients, translating the acute cardioprotective effect of trimetazidine into a more pronounced form. Moreover, the same group of patients had a significantly less eventful follow-up period, with no recorded mortality.

\section{Comparison with other studies}

The cardioprotective anti-ischemic effect of trimetazidine was previously demonstrated in animals $[12,26,29]$ and human beings undergoing PCI [30-33]. Limited studies had evaluated the assumed cardioprotective effects of trimetazidine in patients receiving thrombolytic therapy for STEMI management. This might be due to the well established role of mechanical reperfusion strategy, in addition to wide availability of primary PCI facilities in many countries especially in the last decade. However, in many developing countries especially in rural areas, these facilities are not widely provided and patients' transfer to primary PCI centers, if any, remains costy, time-consuming and sometimes not available. Thrombolytic therapy remains the only reperfusion aid available in these areas. Similar to the present study, a prior study by Di Pasquale et al had previously reported that oral trimetazidine administration shortly before receiving thrombolytic therapy led to earlier peaking and less release of cardiac biochemical markers. Moreover, their study results showed that continued drug administration was associated with less cardiac mortality and favorable LV remodeling process (decreased LVESV) [6]. Another study reported the favorable impact of oral trimetazidine use on ST-segment resolution and creatine kinase release in the same clinical setting [34]. However, these studies were not targeting diabetic patients and the used oral dose of trimetazidine ( $20 \mathrm{mg}$ thrice daily) was different from that used in the current study. Furthermore, their study protocols did not include detailed recording of in-hospital or post-hospitalization adverse events. Similar to the present study, previous studies had reported improvement of LVEF in diabetic patients with ischemic LV systolic dysfunction after regular oral intake of trimetazidine [35-37]. Free radicals are thought to participate in reperfusion arrhythmias that could be detrimental to the prognosis of the patient [38]. A previous study showed a trend in favor of oral trimetazidine (60 $\mathrm{mg}$ on admission then $20 \mathrm{mg}$ for 5 days) in reducing the extent of severe reperfusion arrhythmias [4]. The current study did not reach the same conclusion upon analysis of in-hospital data. However, three patients in the placebo treated group died during the follow-up period raising the possibility of occurrence of malignant ventricular arrhythmias in this group. In contrast to the current study, EMIP-FR study group showed that trimetazidine use in patients treated with thrombolysis was not associated with decreased cardiac mortality after a long-term follow-up period. Trimetazidine receivers also showed higher incidence of in-hospital ventricular arrhythmias, compared to the placebo group [5]. However, EMIP-FR study protocol was different from the one adopted in the current study in many points, for example IV trimetazidine infusion ( $48 \mathrm{~h}$ ) was rather used with no oral doses given thereafter. The authors themselves stated that the regimen used in this study may be inappropriate in terms of dose, site of delivery and/or timing. Also, various types of thrombolytics were used (fibrin and non-fibrin specific), in addition to relatively small percentage (about $12 \%$ of each study arm) of diabetic patients were included. Moreover, assessment of echocardiographic parameters including LV systolic function was not included in the study protocol.

To the best of the author's knowledge, no previous trials addressed the cardioprotective effects of oral trimetazidine in diabetic patients receiving alteplase for management of STEMI. The current study highlighted that less myocardial damage in the acute clinical setting, less eventful 6 months follow-up period and evident recovery of LV systolic function were associated with oral trimetazidine use.

\section{Clinical implications}

Trimetazidine is a well-tolerated drug, widely available in the oral formula. The results of the current study could add benefit to diabetic patients suffering from acute STEMI, commonly encountered by cardiologists in emergency rooms. The author hypothesizes that oral loading with trimetazidine $(70 \mathrm{mg})$ given shortly before thrombolysis in diabetic patients, could help minimize myocardial damage. Additionally, oral daily dosing (35 mg twice daily) of the 
drug could augment the initial cardioprotective effect resulting in improved LV systolic function and less adverse events thereafter.

\section{Limitations of the study}

The results presented in this study only apply for patients defined by inclusion and exclusion criteria. Extended followup (more than 6 months) of myocardial systolic function and adverse events were not included in the current study protocol. The functional aspect of the cardioprotective effect of trimetazidine was not assessed in the current study. This was outside the scope of the study protocol. Large-scale studies are still required to evaluate the long-term functional impact, for example exercise tolerance, of oral trimetazidine administration in similar clinical settings.

\section{Conclusion}

In diabetic patients receiving alteplase (tPA) for management of anterior wall STEMI, oral loading followed by regular daily trimetazidine dosing was associated with less myocardial damage and earlier successful reperfusion, as compared with placebo treatment. Moreover, a 6-month follow-up period showed significant improvement in LV systolic function and less cardiac mortality.

\section{Acknowledgement}

The author likes to express his gratitude for medical, technical and nursing staff of the CCU and the echocardiography laboratory in Cardiology Department of Ain Shams University, for their cooperation to accomplish this work.

\section{Conflict of Interest}

The author declares that there is no conflict of interest regarding publication of this article.

\section{References}

1. Lopaschuk GD. Treating ischemic heart disease by pharmacologically improving cardiac energy metabolism. Am J Cardiol. 1998;82(5A):14K-17K.

2. McClellan KJ, Plosker GL. Trimetazidine. A review of its use in stable angina pectoris and other coronary conditions. Drugs. 1999;58(1):143-157.

3. Steg PG, Grollier G, Gallay P, Morice M, Karrillon GJ, Benamer H, Kempf C, et al. A randomized double-blind trial of intravenous trimetazidine as adjunctive therapy to primary angioplasty for acute myocardial infarction. Int J Cardiol. 2001;77(2-3):263-273.

4. Papadopoulos CL, Kanonidis IE, Kotridis PS, Papayannis IL, Savatis SC, Missopoulou-Kokka AI, Nikolaidis NK, et al. The effect of trimetazidine on reperfusion arrhythmias in acute myocardial infarction. Int J Cardiol. 1996;55(2):137-142.

5. Effect of 48-h intravenous trimetazidine on short- and long-term outcomes of patients with acute myocardial infarction, with and without thrombolytic therapy; A double-blind, placebo-controlled, randomized trial. The EMIP-FR Group. European Myocardial Infarction Project--Free Radicals. Eur Heart J. 2000;21(18):1537-1546.

6. Di Pasquale P, Lo verso P, Bucca V, Cannizzaro S, Scalzo S, Maringhini G, Rizzo R, et al. Effects of trimetazidine administration before thrombolysis in patients with anterior myocardial infarction: short-term and long-term results. Cardiovasc Drug Ther. 1999;13:423-428.

7. Clauser P, Harpey C. Antioxidant properties of an antiischaemic agent: trimetazidine. Adv Exp Med Biol. 1990;264:247-250.

8. Leizorovicz A, Boissel JP, Robert F. Coronary reperfusion rates in acute myocardial infarction patients after thrombolytic treatment with anistreplase: correlation with the delay from onset of symptoms to treatment: a review of 424 case records of patients admitted to coronary reperfusion studies with anistreplase. J Cardiovasc Pharmacol. 1992;19(1):34-39.

9. Elimadi A, Settaf A, Morin D, Sapena R, Lamchouri F, Cherrah Y, Tillement JP. Trimetazidine counteracts the hepatic injury associated with ischemia-reperfusion by preserving mitochondrial function. J Pharmacol Exp Ther. 1998;286(1):23-28.

10. Aubert A, Bernard C, Clauser P, Harpey C, Vaudry H. Effect of phenazine methosulfate on electrophysiological activity of the semicircular canal: antioxidant properties of trimetazidine. Eur J Pharmacol. 1989;174(23):215-225.

11. Kantor PF, Lucien A, Kozak R, Lopaschuk GD. The antianginal drug trimetazidine shifts cardiac energy metabolism from fatty acid oxidation to glucose oxidation by inhibiting mitochondrial long-chain 3-ketoacyl coenzyme A thiolase. Circ Res. 2000;86(5):580-588.

12. Lavanchy N, Martin J, Rossi A. Anti-ischemic effects of trimetazidine: 31P-NMR spectroscopy in the isolated rat heart. Arch Int Pharmacodyn Ther. 1987;286(1):97-110.

13. Questions and answers on the review of medicines containing trimetazidine $(20 \mathrm{mg}$ tablets, $35 \mathrm{mg}$ modified release tablet and $20 \mathrm{mg} / \mathrm{ml}$ oral solution). http://www. ema.europa.eu/docs/en_GB/document_library/Referrals_document/Trimetazidine_31/WC500129195.pdf.

14. Task Force on the management of STseamiotESoC, Steg PG, James SK, Atar D, Badano LP, BlomstromLundqvist C, Borger MA, et al. ESC Guidelines for the 
management of acute myocardial infarction in patients presenting with ST-segment elevation. Eur Heart J. 2012;33(20):2569-2619.

15. Mancia G, Fagard R, Narkiewicz K, Redon J, Zanchetti A, Bohm M, Christiaens T, et al. 2013 ESH/ESC guidelines for the management of arterial hypertension: the Task Force for the Management of Arterial Hypertension of the European Society of Hypertension (ESH) and of the European Society of Cardiology (ESC). Eur Heart J. 2013;34(28):2159-2219.

16. Expert Panel on Detection E, Treatment of High Blood Cholesterol in A. Executive Summary of The Third Report of The National Cholesterol Education Program (NCEP) Expert Panel on Detection, Evaluation, And Treatment of High Blood Cholesterol In Adults (Adult Treatment Panel III). JAMA. 2001;285(19):2486-2497.

17. Cerqueira MD, Weissman NJ, Dilsizian V, Jacobs AK, Kaul S, Laskey WK, Pennell DJ, et al. Standardized myocardial segmentation and nomenclature for tomographic imaging of the heart. A statement for healthcare professionals from the Cardiac Imaging Committee of the Council on Clinical Cardiology of the American Heart Association. Circulation. 2002;105(4):539-542.

18. Chaudhry FA, Singh B, Galatro K. Reversible left ventricular dysfunction. Echocardiography. 2000;17(5):495506.

19. Meluzin J, Cerny J, Frelich M, Stetka F, Spinarova L, Popelova J, Stipal R. Prognostic value of the amount of dysfunctional but viable myocardium in revascularized patients with coronary artery disease and left ventricular dysfunction. Investigators of this Multicenter Study. J Am Coll Cardiol. 1998;32(4):912-920.

20. de Lemos JA, Antman EM, Giugliano RP, McCabe CH, Murphy SA, Van de Werf F, Gibson CM, et al. ST-segment resolution and infarct-related artery patency and flow after thrombolytic therapy. Thrombolysis in Myocardial Infarction (TIMI) 14 investigators. Am J Cardiol. 2000;85(3):299-304

21. Pornin M, Harpey C, Allal J, Sellier P, Ourbak P. Lack of effects of trimetazidine on systemic hemodynamics in patients with coronary artery disease: a placebo-controlled study. Clin Trials Metaanal. 1994;29(1):49-56.

22. Lopaschuk GD, Barr R, Thomas PD, Dyck JR. Beneficial effects of trimetazidine in ex vivo working ischemic hearts are due to a stimulation of glucose oxidation secondary to inhibition of long-chain 3-ketoacyl coenzyme a thiolase. Circ Res. 2003;93(3):e33-37.

23. Morin D, Elimadi A, Sapena R, Crevat A, Carrupt PA, Testa B, Tillement JP. Evidence for the existence of $[3 \mathrm{H}]$-trimetazidine binding sites involved in the regulation of the mitochondrial permeability transition pore. Br J Pharmacol. 1998;123(7):1385-1394.

24. Grekas D, Dioudis C, Papageorgiou G, Iliadis S, Zilidis C, Alivanis P, Dimitriadou A, et al. Lipid peroxidation after acute renal ischemia and reperfusion in rats: the effect of trimetazidine. Ren Fail. 1996;18(4):545-552.

25. Ozden A, Aybek Z, Saydam N, Calli N, Saydam O, Duzcan E, Guner G. Cytoprotective effect of trimetazidine on $75 \mathrm{~min}$ warm renal ischemia-reperfusion injury in rats. Eur Surg Res. 1998;30(4):227-234.

26. Tsimoyiannis EC, Moutesidou KJ, Moschos CM, Karayianni M, Karkabounas S, Kotoulas OB. Trimetazidine for prevention of hepatic injury induced by ischaemia and reperfusion in rats. Eur J Surg. 1993;159(2):89-93.

27. Noble MI, Belcher PR, Drake-Holland AJ. Limitation of infarct size by trimetazidine in the rabbit. Am J Cardiol. 1995;76(6):41B-44B.

28. Argaud L, Gomez L, Gateau-Roesch O, Couture-Lepetit E, Loufouat J, Robert D, Ovize M. Trimetazidine inhibits mitochondrial permeability transition pore opening and prevents lethal ischemia-reperfusion injury. $\mathrm{J}$ Mol Cell Cardiol. 2005;39(6):893-899.

29. Rahman F, Toshima Y, Kohno H, Kinoshita K, Tokunaga $\mathrm{K}$. The protective effects of trimetazidine on normothermic ischemic myocardium in rats. Jpn J Surg. 1989;19(3):346-350.

30. Kober G, Buck T, Sievert H, Vallbracht C. Myocardial protection during percutaneous transluminal coronary angioplasty: effects of trimetazidine. Eur Heart J. 1992;13(8):1109-1115.

31. Polonski L, Dec I, Wojnar R, Wilczek K. Trimetazidine limits the effects of myocardial ischaemia during percutaneous coronary angioplasty. Curr Med Res Opin. 2002;18(7):389-396.

32. Bonello L, Sbragia P, Amabile N, Com O, Pierre SV, Levy S, Paganelli F. Protective effect of an acute oral loading dose of trimetazidine on myocardial injury following percutaneous coronary intervention. Heart. 2007;93(6):703-707.

33. Labrou A, Giannoglou G, Zioutas D, Fragakis N, Katsaris G, Louridas G. Trimetazidine administration minimizes myocardial damage and improves left ventricular function after percutaneous coronary intervention. Am J Cardiovasc Drugs. 2007;7(2):143-150.

34. Rebrova TY, Lasukova TV, Afanas'ev SA, Perchatkin VA, Maksimov IV, Markov VA. Cardioprotective effect of trimetazidine during thrombolytic therapy in patients with acute myocardial infarction. Bull Exp Biol Med. 2002;134(6):559-561.

35. Belardinelli R, Cianci G, Gigli M, Mazzanti M, Lacalaprice $\mathrm{F}$. Effects of trimetazidine on myocardial perfusion and left ventricular systolic function in type 2 diabetic patients with ischemic cardiomyopathy. J Cardiovasc Pharmacol. 2008;51(6):611-615.

36. Thrainsdottir IS, von Bibra H, Malmberg K, Ryden L. Effects of trimetazidine on left ventricular function in patients with type 2 diabetes and heart failure. J Cardiovasc Pharmacol. 2004;44(1):101-108. 
37. Fragasso G, Piatti Md PM, Monti L, Palloshi A, Setola E, Puccetti P, Calori G, et al. Short- and long-term beneficial effects of trimetazidine in patients with diabetes and ischemic cardiomyopathy. Am Heart J.
2003;146(5):E18.

38. Burton KP. Evidence of direct toxic effects of free radicals on the myocardium. Free Radic Biol Med. 1988;4(1):15-24. 